\title{
Hierarchical Optimization Model Based on V2G Technology
}

\author{
Wei LUO, Xiaohu ZHU1', Liansong YU \\ State Grid Electric Power Research Institute, Wuhan Nari Limited Liability \\ Company, Wuhan 430074, China
}

\begin{abstract}
Large-scale electric vehicle (EV) random access to the power grid, the load peak-valley difference will become larger, seriously affect the stable operation of the power grid. In this paper, a V2G based bi-level optimal scheduling model for EV charging and discharging is proposed. The upper model takes the minimum variance of the total load as the objective function, and the lower model takes the increase of user participation and the maximization of user revenue as the objective function. The multi-population genetic algorithm is used to analyze the model, and the results show that the model can not only smooth the load fluctuation, effectively reduce the load peak-valley difference, but also maximize the economic benefits of users participating in $\mathrm{V} 2 \mathrm{G}$ service.
\end{abstract}

Keywords. Power grid load, double-layer model, load peak-valley, genetic algorithm, user income.

\section{Introduction}

Compared with traditional fuel vehicles, electric vehicles have great advantages in energy saving and emission reduction, and in achieving green travel, it is an inevitable trend that electric vehicles will be chosen as the way of travel in the future[1,2].Electric vehicles are energy storage devices that can be plugged into the grid as a load or as a distributed power device, and can also be used to enhance the efficient energy use through the two way interaction of the grid. The reasonable dispatch of electric vehicle load can improve the operation state of the power grid, ease the power load in the power grid, effectively manage the charging load, realize load peak shaving and valley filling, at the same time, cooperate with the access of new energy sources such as photovoltaic and wind energy, we will accelerate the integration of wind, light and storage of new energy sources[3,4].Therefore, it is very important to optimize the charging and discharging strategy of EV.

In order to solve the problem of power load mutation and multi-dimension treatment of centralized dispatching mode caused by large-scale electric vehicles (evs) accessing to power grid, regional hierarchical control is an effective means to guide large-scale electric vehicles (evs) to control[5-7]. The model established in the above-mentioned literatures is mainly aimed at the benefit of power grid measurement, and the ultimate goal is to smooth the load fluctuation, but it does not take into account the response of users and the ability of regional electric vehicles to participate in dispatching. Reference

\footnotetext{
${ }^{1}$ Corresponding Author; Email: jzzxh18@163.com
} 
[8-11] by defining the loss cost and the average discharge index in the charging process, consummating the user side demand, establishing the grid side and the user side charging and discharging strategy. The model in reference [12] mentioned that the upper layer aims to minimize the fluctuation of the load curve, and the lower layer aims to minimize the distribution loss of the power plant and the cost of materials such as coal, power grid measurement and customer demand imbalance. In reference [13-16], a particle swarm optimization (PSO-RRB) algorithm is proposed to model the charging power limit without considering the different demands of users.

According to the above research situation, this paper takes a residential area as the research object, comprehensively considers the relationship among dispatch center, regional agent, and users, a dual-layer charge-discharge scheduling model for electric vehicles (evs) is proposed, which takes into account the willingness and capability of the users. The upper model minimizes the total load variance of the grid system by determining the charging load and discharging power in each period, and the lower model is based on whether the users have the willingness to participate and the dispatching ability of the users, under the premise of the cooperation of the regional agent with the dispatch center plan, it is important to increase the user participation and maximize the user income. In this paper, the ordered charging model is analyzed by using multi-population genetic optimization algorithm, and the optimal power distribution based on the user's scheduling intention and ability is obtained, it provides a basis for electric vehicle charging and discharging scheduling strategy.

\section{Layered and optimized architecture for electric vehicles}

\subsection{Hierarchical Scheduling architecture design}

The idea of hierarchical dispatch is to stratify the dispatch area according to the voltage level, and then divide the dispatch area according to the region. The user communicates with the regional agent, reports his dispatch intention and ability, the agent collects the regional power demand and evaluates the regional dispatch ability, and feeds back the information to the system dispatch center. The hierarchical scheduling architecture constructed in this article is shown in Figure 1.

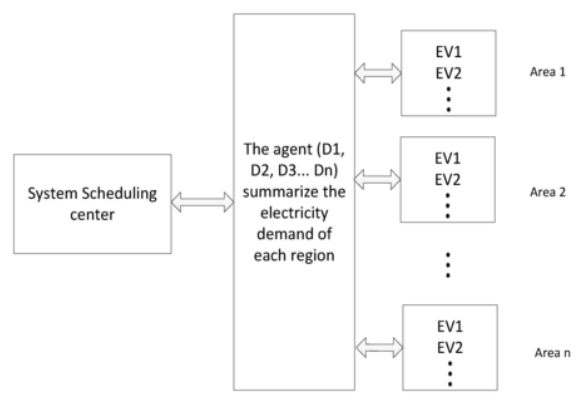

Figure 1. Hierarchical Scheduling Architecture Model for EVs

\subsection{Charging and discharging load analysis of electric vehicles}

The research on the trip law of electric vehicle is the basis of the implementation of the dispatching strategy. When the residents in the urban community use the fuel car or electric vehicle, it has little influence on the trip characteristics. This article is based on 
the 2009 U.S. household car travel survey [17-20] and the following is the probability density function of when electric cars go home and leave home.Assuming that the owner participates in the dispatching plan immediately after returning home, that is, the ev is immediately connected to the power grid, the time when EV i starts to connect to the power grid is $\operatorname{tg}, \mathrm{i} \sim \mathrm{N}(17.9,3.42)$, and the time when EV I is expected to leave the power grid is $\mathrm{tl}, \mathrm{i} \sim \mathrm{N}(9.24,3.162)$.

$$
f_{d}(x)=\frac{1}{x \sigma \sqrt{2 \pi}} \exp \left[\frac{-(\ln x-\mu)^{2}}{2 \sigma^{2}}\right]
$$

In this paper, the hierarchical architecture is simulated based on the above probability distribution model, and the electric vehicle (EV) is investigated and sampled by Monte Carlo sampling method [21], thus, the charging load of EV is modeled to optimize the parameters of the model.

\section{Electric vehicle response capability based on V2G}

For electric vehicles in the V2G program, the battery capacity can be divided into the battery capacity B1 for the V2G program, the battery capacity B2 for daily driving, and the battery capacity B3 for standby driving, as shown in Figure 2.

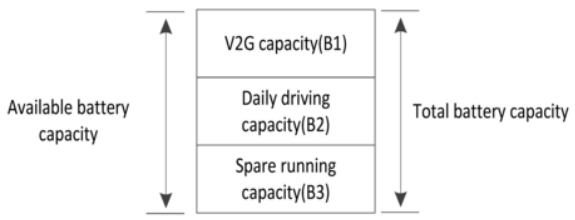

Figure 2. Electric vehicle battery capacity division

The V2G response capacity of a single vehicle can be reported according to the capacity of the B1 when the battery capacity of the B1 and B2 are satisfied when the EV is recharged, the responsiveness of electric vehicles should also be related to the start and end of charging and the minimum time for the maximum power to reach the desired battery state, while the responsiveness of users is also a key factor in the responsiveness.

If the value of response capability is $\mathrm{K}$, Where, $T_{l, i}$ represents the time when the electric vehicle leaves the grid, $T_{g, i}$ represents the time when the electric vehicle is connected to the grid, and $T_{\text {mini, }}$ represents the shortest time when the maximum power reaches the expected battery state. When $\mathrm{K}>1$, the electric vehicle has the response capability. The response capacity of ev is also related to its battery capacity Q, so the formula is as follows:

$$
k=\frac{\left(T_{l, i}-T_{g, i}\right) Q}{T_{\min , i}}
$$




\section{Charging and discharging scheduling strategy of electric vehicles based on double-layer optimization model}

\subsection{Two-level optimization model}

In this paper, combined with the travel characteristics of residential areas, the overall power consumption strategy in each area is formulated by the upper dispatch center, and the regional agent makes the overall power consumption strategy in the area under the overall power consumption strategy, so that the total fluctuation curve of the system during the charging and discharging period is minimized, at the same time, the agent's actual dispatch and the dispatch center's power consumption strategy deviation sum is minimum. In the underlying model, the regional agent manages the pre-scheduled charging of the electric vehicles in the region, and maximizes the revenue of the users by combining the users'scheduling willingness and ability.

\subsection{Upper objective function}

The upper objective function is:

$$
\min F=\frac{1}{T-1} \sum_{t=1}^{T}\left(P_{d, t}+\sum_{n=1}^{N_{0}} P_{n, t}-P_{D E}-\bar{P}_{a}\right)^{2}+\alpha \sum_{N=1}^{N_{0}} \sum_{t=1}^{T}\left|P D_{n, t}-P_{n, t}\right|
$$

Where T represents 24 time periods in a day; $\mathrm{N}$ is the number of agents; $P_{d, t}$ is the load of non-electric vehicle in t period; $P_{n, t}$ represents the charge and discharge load of electric vehicles, and N0 represents the total number of agents; $\mathrm{P}_{\mathrm{DE}}$ represents the output of other distributed energy sources in the region; $\bar{P}_{a}$ represents the average load of the system within T periods, $\overline{P_{a}}=\frac{1}{T} \sum_{t=1}^{T}\left(P_{d, t}+\sum_{n=1}^{N_{0}} P_{n, t}-P_{D E}\right)$, The scheduling deviation function between the dispatching center and regional agents is: $F=\alpha \sum_{N=1}^{N_{0}} \sum_{t=1}^{T}\left|P D_{n, t}-P_{n, t}\right|$, Where $\alpha$ represents the penalty coefficient of violation and represents the scheduling deviation and constraints of regional agents; $P D_{n, t}$ represents the scheduling plan made by the regional agent.

\subsection{Lower level objective function}

For users, the purpose of participating in the V2G scheduling plan is to reduce charging costs and travel costs, and users can obtain maximum benefits according to their response ability.

The lower level objective function with the minimum charging cost is as follows:

$$
\min f=\left(\sum_{T_{0}}^{T_{l}} \overline{P_{c, i}} C_{c, i} a-\sum_{T_{0}}^{T_{l}} \overline{P_{d, i}} C_{d, i} b\right)\left(T_{l}-T_{0}\right) \overline{K_{i}}
$$

$T_{0}$ is the starting time of charging and discharging, $T_{l}$ is the ending time of charging and discharging, $\overline{P_{c, i}}$ and $\overline{P_{d, i}}$ are the charging power and discharging power respectively, $C_{c, i}$ and $C_{d, i}$ are the charging price and discharging price respectively, 
and $\mathrm{a}, \mathrm{b}$ means charge parameter and discharge parameter respectively, when charging, $\mathrm{a}$ is $1, \mathrm{~b}$ is 0 , when discharging, $\mathrm{a}$ is $0, \mathrm{~B}$ is 1 . It means the average response ability of $\overline{K_{i}}$ in the area under the jurisdiction of the agent, the average response ability of different area under the jurisdiction of the agent is different.

\section{Model solving method}

There are many constraints in the bi-level optimization model of electric vehicle charging and discharging, but due to the evolution of genetic algorithm (SGA), it is not necessary to pay much attention to whether the constraints are linear or nonlinear, but directly to the objective function as the search information, with a very high degree of flexibility. When there are super-strong individuals in the group or the group size is small, the convergence boundary will move forward $[22,23]$. In this paper, a multi-population genetic optimization algorithm (MPGA) is used, and different parameters are set for different populations, and immigration operator is introduced to achieve the simultaneous evolution of different populations. Finally, the elite population is selected [24-26]. Based on the bi-level optimization model of electric vehicle charging and discharging, the optimal charging power of each period can be calculated by multipopulation genetic optimization algorithm.

The implementation process of multi-population genetic optimization algorithm is to select the best individuals and put them into elite population each time the population evolves, and the elite population is selected separately and does not participate in the evolution until the elite population reaches the limit and then terminates the program.

\section{Case analysis}

In this paper, 5 large-scale communities with 5 district agents as an example, agents in coordination with the General Scheduling Center Plan, responsible for their own district charge and discharge scheduling. Set the jurisdiction of the electric vehicles are ordinary household electric vehicles, and electric vehicles are generally charged at night. Let's say an EV in one area responds to $90 \%$ of V2G. Select the base power of $200 \mathrm{kVA}$, the scheduling cycle will be 0 to 24 points, to hours as time interval units, a total of 24 time periods. That's 24 hours. In the current period, assume that the electric vehicle chargedischarge load and other basic load does not change, and the owner of the average monthly charge times is 10 times.

The number of EVs and the average V2G capability of each agent in the jurisdiction are set as shown in Table 1. Ev parameters in Table 2.

Table 1. The number and average response capacity of electric vehicles in each agent's jurisdiction area

\begin{tabular}{ccc}
\hline Area & Number of EV & Average response capacity \\
\hline Area1 & 2800 & 1.26 \\
Area2 & 3300 & 1.34 \\
Area3 & 3800 & 1.41 \\
Area4 & 4300 & 1.49 \\
Area5 & 4800 & 1.53 \\
\hline
\end{tabular}


Table 2. Electric vehicle parameter list

\begin{tabular}{lc}
\hline \multicolumn{1}{c}{ Parameter } & Value \\
\hline Average charging efficiency $\%$ & 90 \\
Average discharge efficiency $/ \%$ & 90 \\
Total energy stored in battery $/ \mathrm{kWh}$ & 120 \\
The user wants the amount of charging $/ \%$ & 90 \\
Power consumption for 100 kilometerskWh $/ 100 \mathrm{~km}$ & 13 \\
\hline
\end{tabular}

The V2G response optimization results of the EV are shown in Figure 3., the change of power load can be seen under the condition of disordered charging and under the optimal strategy. The peak-to-peak load difference is $272.33 \mathrm{kw}$ between 9:00 10:00,11:00 12:00 and 18:00 21:00. After the optimized scheduling strategy adopted in this paper, the peak load appears at 9:00 11:00,20:00 21:00. The difference between peak load and valley load is $90.21 \mathrm{kw}$, which can reduce the fluctuation of load compared with the state of disordered charging.

As can be seen from Figure 4, the optimization strategy is related to the number of regional electric vehicles and increases with the number of regional electric vehicles. The maximum peak-valley difference is $46 \mathrm{KW}$ in area 1 and $47.1 \mathrm{kw}$ in area 5 . The trend of electric load is the same, and the average difference between peak and peak is $11 \mathrm{KW}$, and the average difference between valley and valley is $19.8 \mathrm{KW}$.

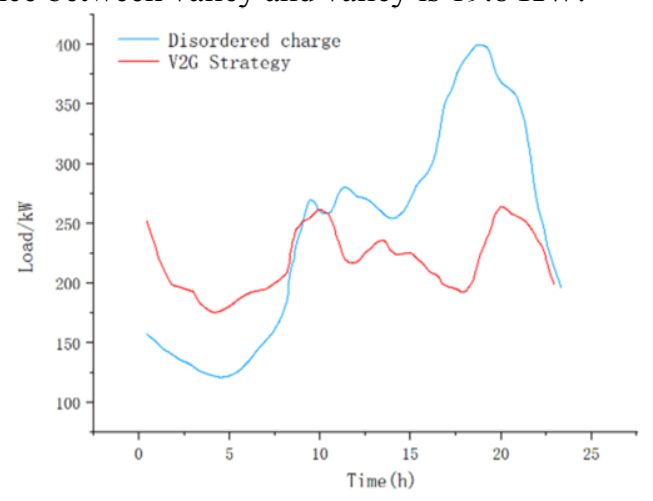

Figure 3.V2G Response Optimization Curve of EVs

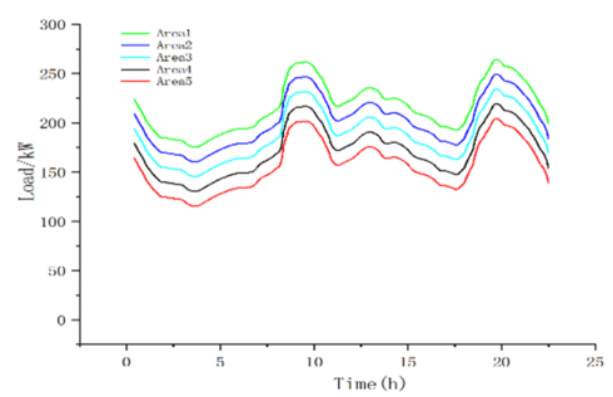

Figure 4. Load curves of each region under the optimization strategy 


\section{Conclusion}

In this paper, an optimal scheduling strategy for EV charging and discharging in V2G mode is proposed. The objective of the upper model is to minimize the variance of the total load fluctuation of the power grid system, and the lower model is based on whether the users have the willingness to participate and the dispatching ability of the users, under the premise that the regional agents cooperate with the dispatching center planning, pay attention to the balance of regional power load. The model is analyzed by using group genetic algorithm. The results show that the model can smooth the load fluctuation, reduce the load peak-valley difference and stabilize the regional load.

\section{References}

[1] Cai Jiayu, Zhao zhigang. A Review of The Interaction Between Electric Vehicles and Power Grid in V2G Mode [J]. Electronics World,2021(07):19-20.

[2] Li Yirang, Zhangshu,Xiao Xianyong,et al.The charging and discharging scheduling strategy for electric vehicles in V2G mode considering demand from both sides [J]. Electric Power Automation Equipment, 2021,41(03):129-135+143.

[3] Ruifeng Shi, Shaopeng Li, Penghui Zhang, Kwang Y. Lee. Integration of renewable energy sources and electric vehicles in V2G network with adjustable robust optimization[J]. Renewable Energy,2020,153.

[4] Schetinger Annelys Machado, Dias Daniel Henrique Nogueira, Borba Bruno Soares Moreira Cé sar,Pimentel da Silva Gardenio Diogo. Techno - economic feasibility study on electric vehicle and renewable energy integration: A case study[J]. Energy Storage,2020,2(6).

[5] Popoola A. I, Akinpelu E. O, Ewetumo T. Development and Performance Evaluation of an Intelligent Electric Power Switching System[J]. Journal of Trend in Scientific Research and Development,2021,5(3).

[6] Yu. M. In'kov,E. N. Rozenberg,A. I. Maron. Simulation of the Process of Implementation of an Intelligent Electric Power Metering System[J]. Russian Electrical Engineering,2020,91(1).

[7] Evgeny Tretyakov. Demand management by active consumers in intelligent electric power systems[J]. E3S Web of Conferences,2020,157.

[8] Huang Minfa, Xiang Baolin, Peng Juanjuan, Zhang Lu. Optimal Configuration Method for EV Charging Station in Distribution Network Considering User Adjustment under V2G mode[J]. IOP Conference Series: Earth and Environmental Science,2021,784(1).

[9] Heilmann C., Friedl G.. Factors influencing the economic success of grid-to-vehicle and vehicle-to-grid applications-A review and meta-analysis[J]. Renewable and Sustainable Energy Reviews,2021,145.

[10] Tan Kang Miao, Ramachandaramurthy Vigna K.,Yong Jia Ying,Tariq Mohd. Experimental verification of a flexible vehicle-to-grid charger for power grid load variance reduction[J]. Energy,2021,228.

[11] Popoola A. I, Akinpelu E. O, Ewetumo T. Development and Performance Evaluation of an Intelligent Electric Power Switching System[J]. Journal of Trend in Scientific Research and Development,2021,5(3).

[12] Wu Wei, Lin Boqiang. Benefits of electric vehicles integrating into power grid[J]. Energy,2021,224.

[13] Zheng Xue qin, Yao Yi ping. Multi-objective capacity allocation optimization method of photovoltaic EV charging station considering V2G[J]. Journal of Central South University,2021,28(2).

[14] Liu Lijun, Xie Feng, Huang Zonglong, Wang Mengqi. Multi-Objective Coordinated Optimal Allocation of DG and EVCSs Based on the V2G Mode[J]. Processes,2020,9(1).

[15] Wang Dian, Sechilariu Manuela,Locment Fabrice. PV-Powered Charging Station for Electric Vehicles: Power Management with Integrated V2G[J]. Applied Sciences,2020,10(18).

[16] Shigeru Tamura. A V2G strategy to increase the cost-benefit of primary frequency regulation considering EV battery degradation[J]. Electrical Engineering in Japan,2020,212(1-4).

[17] Hao Tang, Chang Liu,Yonglun Cao,Kai Lv,Qianli Zhang. Hierarchical scheduling learning optimisation of two-area active distribution system considering peak shaving demand of power grid[J]. Discrete Event Dynamic Systems,2021(prepublish).

[18] Ruifeng Shi, Shaopeng Li, Penghui Zhang, Kwang Y. Lee. Integration of renewable energy sources and electric vehicles in V2G network with adjustable robust optimization[J]. Renewable Energy,2020,153.

[19] Yu. M. In'kov, E. N. Rozenberg,A. I. Maron. Simulation of the Process of Implementation of an Intelligent Electric Power Metering System[J]. Russian Electrical Engineering,2020,91(1).

[20] Evgeny Tretyakov. Demand management by active consumers in intelligent electric power systems[J]. E3S Web of Conferences,2020,157. 
[21] He Wei, Chen Fan, Miao Han, et al.Cross-entropy Monte Carlo Method for Power System Abundance Evaluation [J].Acta electronica sinica,2020,35(03):235-245.

[22] Li Zening, Su Su, Jin Xiaolong, Chen Houhe, Li Yujing, Zhang Renzun. A hierarchical scheduling method of active distribution network considering flexible loads in office buildings[J].International Journal of Electrical Power and Energy Systems,2021,131.

[23] Shen Xiaoyang. A hierarchical task scheduling strategy in mobile edge computing[J]. Internet Technology Letters,2020,4(5).

[24] Li Jiale, Liu Zhenbo, Wang Xuefei. Public charging station location determination for electric ridehailing vehicles based on an improved genetic algorithm[J]. Sustainable Cities and Society,2021,74.

[25] Jordán Jaume, Palanca Javier,del Val Elena, Julian Vicente, Botti Vicent. Localization of charging stations for electric vehicles using genetic algorithms[J]. Neurocomputing,2021,452.

[26] Wang Jianjun, Pan Junnan, Huo Jikun, Wang Ran, Li Li, Nian Tiantian. Research on Optimization of Multi-AGV Path Based on Genetic Algorithm Considering Charge Utilization[J]. Journal of Physics: Conference Series,2021,1769(1). 